\title{
Aplicación de la Activación Conductual en un Paciente con Sintomatología Depresiva
}

\section{Behavioral Activation Intervention in a Patient With Depressive Symtomatology}

\author{
Jorge Barraca Mairal \\ Universidad Camilo José Cela
}

\begin{abstract}
Resumen. Se presenta el tratamiento de un sujeto varón de 44 años con síntomas de depresión. La intervención se organizó a partir del análisis propuesto por la Activación Conductual (AC), que contempla algunos tipos de depresión como formas elaboradas de evitación. Entre los componentes del tratamiento se incluyó la recuperación de tareas abandonadas, el replanteamiento de nuevos objetivos vitales, la extinción de conductas de evitación, la programación horaria y la recuperación de obligaciones domésticas, laborales y sociales. Después de siete meses de intervención se apreciaron cambios significativos en las medidas pretratamiento (BDI-II, AAQ, ATQ, EROS y BADS), así como en el número y el tipo de actividades desarrolladas. Tras este tiempo el cuadro depresivo podía considerarse remitido y el paciente había recuperado un trabajo productivo. Los beneficios terapéuticos se mantuvieron durante los siguientes cinco meses de seguimiento. A pesar de la gravedad del cuadro, el sujeto no deseaba tomar medicación antidepresiva, por lo que ésta no se empleó en ningún momento. Los buenos resultados obtenidos no obstante esta circunstancia y la duración relativamente breve de la intervención suponen un aval importante para el modelo de terapia propuesto por la AC para este tipo de casos.
\end{abstract}

Palabras clave: activación conductual, depresión, estudios de casos, experimento de caso único.

\begin{abstract}
Treatment for a 44-year-old man with depressive symtomatology is set out. The intervention proposed was based on the behavioral activation (BA) analysis, that sees some types of depression as elaborated avoidance modalities. Treatment components included recuperation of tasks that had previously been given up, reconsideration of life goals, ending of avoidance behaviors, time scheduling and reengagement with housework, labour duties and social responsibilities. After seven months of treatment remarkable changes appeared in the pre-treatment measures (BDI-II, AAQ, ATQ, EROS, and BADS), as well as in the number and kind of activities carried out. Depression was in full remission and the patient had regained a state of productive work. Follow-up showed that therapeutic benefits persisted for the next five months. In spite of the severity of the set of symptoms, the patient did not want to take antidepressant medication, and so it was never used. The good outcomes obtained in spite of this circumstance and despite the relatively short length of time of intervention strongly support the model proposed by BA for these kinds of cases.
\end{abstract}

Key words: behavioral activation, eepression, case studies, single-case experiment.

La correspondencia sobre este artículo dirigirla al autor al e-mail:

jbarraca@ucjc.edu 


\section{Introducción}

En los últimos años, las intervenciones con métodos de activación conductual encaminadas a paliar los síntomas depresivos - como, por ejemplo, la jerarquización de tareas, la programación de objetivos vitales, el reforzamiento de conductas de contacto social o la realización de actividades gratificantes -, han recuperado el interés de los clínicos (Kanter, Busch y Rusch, 2009; Lejuez, Hopko y Hopko, 2002; Martell, Addis y Jacobson, 2001). En realidad, estas estrategias nunca dejaron de tener cabida en los casos más graves, pero se consideraban un paso inicial y preparatorio para el empleo de las técnicas cognitivas que, según se creía, acogían los procedimientos que posibilitaban mejorar del cuadro depresivo de forma estable y evitar futuras recaídas (Beck, Rush, Shaw y Emery, 1979). Sin embargo, las actuales revisiones metaanalíticas (Cuijpers, van Straten y Warmer-dam, 2007) y los trabajos experimentales en que se han comparado distintos acercamientos terapéuticos medicación antidepresiva, terapia cognitiva y terapia de activación conductual- (Dimidjian et al., 2006) han revelado que las intervenciones puramente conductuales son suficientes para un abordaje eficiente $\mathrm{y}$ eficaz en los cuadros depresivos, incluso teniendo en cuenta el largo plazo (Dobson et al., 2008)

A la par que se estudiaban sistemáticamente los componentes realmente útiles de la terapia cognitiva (Gortner, Gollan, Dobson y Jacobson, 1998; Jacobson et al., 1996), se formalizaba un acercamiento a la clínica del trastorno depresivo que incorporaba un análisis de sus factores de mantenimiento. En él se recuperaban los modelos explicativos de Ferster (1973) y del primer Lewinsohn (1974), pero se ampliaban, y se enfatizaba el carácter funcional que debe regir todo el análisis del caso (Hopko, Lejuez, Ruggiero y Eifert, 2003; Jacobson y Gortner, 2000; Jacobson, Martell y Dimidjian, 2001). De este modo, lo que ha acabado surgiendo no es una mera actualización de las técnicas de activación conductual, sino toda una terapia (la Activación Conductual, AC), que, de forma sencilla, podría definirse como un procedimiento bien estructurado, aunque flexible y con un carácter ideográfico, encaminado a lograr que los pacientes deprimidos aprendan a organizar sus vidas y cambiar su entorno para que se restablezca su contacto con fuentes de reforzamiento positivo. Por este énfasis en el análisis funcional y por el planteamiento contextual - y en parte también por alguna de las técnicas que incorpora - la $\mathrm{AC}$ ha venido a encuadrarse dentro de la denominada tercera generación de la corriente cognitivo-conductual (Barraca, 2006; Hayes, 2004; Pérez-Álvarez, 2006).

Hoy por hoy, la AC ha logrado una amplia evidencia de su eficacia, tanto en trabajos experimentales bien controlados (Dimidjian et al., 2006; Gawrysiak, Nicholas y Hopko, 2009; Hopko Lejuez, LePage, Hopko y McNeil, 2003; Pagoto, Bodenlos, Schneider, Olendzki, Spates y Ma, 2008), como en estudios de caso (Bottonari, Roberts, Thomas y Read, 2008; Hopko, Lejuez y Hopko, 2004; Hopko, Robertson y Lejuez, 2006; Santiago-Rivera, Kanter, Benson, Derose, Illes y Reyes, 2007), y se ha incorporado en los manuales más reputados de tratamiento psicológico (cf. Dimidjian, Martell, Addis y Herman-Dunn, 2008).

No obstante, un problema que condiciona negativamente la difusión y el desarrollo de la AC como terapia consiste en la existencia de dos protocolos distintos de intervención: el de la Activación Conductual (AC) (Jacobson et al., 2001; Martell et al., 2001; Martell, Dimidjian y Herman-Dunn, 2010) y el de la Terapia de Activación Conductual para la Depresión (TACD) (Lejuez, Hopko y Hopko, 2001; Lejuez et al., 2002; Lejuez, Hopko, LePage, Hopko y McNeil, 2001). Si bien ambas intervenciones coinciden en los aspectos esenciales (automonitorización del estado de ánimo, jerarquización de tareas, establecimiento de metas concretas, etc.) también son apreciables entre ellas algunas diferencias (duración de la intervención, estructuración de las sesiones, énfasis en el reforzamiento positivo o en el negativo, etc.) (Cfr. para una revisión detallada Barraca, 2009 o Hopko et al., 2003). Aunque la coexistencia de los dos protocolos produce una imagen de dispersión y genera dudas para el clínico que desea contar con guías de actuación unificadas y bien contrastadas, es probable que los actuales esfuerzos por integrar ambos procedimientos (como, por ejemplo, supone el ma- 
nual de Kanter et al., 2009) solventen este inconveniente.

Aunque la AC se ha empleado con población latina (Santiago-Rivera et al., 2007), no existe aún una bibliografía que facilite su conocimiento entre los psicólogos clínicos hispanohablantes. Además, tampoco se han publicado en España ejemplos de casos clínicos que complementen los trabajos teóricos con los que ya se cuenta (Barraca, 2009; Pérez-Álvarez, 2008) o los referidos a instrumentos de evaluación de la AC (Barraca y Pérez-Álvarez, pendiente de publicación).

El presente artículo tiene como objetivo cubrir esta laguna al ofrecer de forma detallada una intervención llevada a cabo desde la $\mathrm{AC}$ en un sujeto con síntomas depresivos. El formato de presentación seguido aquí para la exposición se acoge a las recomendaciones sugeridas por Virués-Ortega y Moreno-Rodríguez (2008) para la publicación de informes de casos clínicos en psicología conductual.

\section{Presentación del caso}

\section{Identificación del paciente y motivo de consulta}

El paciente es un varón de 44 años, casado y con dos hijos. Aunque abogado de formación, en la actualidad y desde hace unos años, vive exclusivamente de su actividad intelectual (libros, conferencias, colaboraciones en medios de comunicación, etc.). No tiene antecedentes familiares de enfermedad mental. Refiere un breve episodio ansioso, sucedido unos veinte años antes, que remitió rápidamente tras ser tratado con ansiolíticos.

Pocas semanas antes de acudir a consulta, confiesa a su mujer una relación extramatrimonial. Empieza entonces un periodo de difícil convivencia, en el que ambos valoran distintas posibilidades y por el que el paciente comienza la terapia. Finalmente, un par de meses después, su mujer decide divorciarse. A partir de ese momento, y pese a que inicialmente parece más aliviado, empieza a sufrir un profundo malestar, exhibe una conducta más pasiva y su trabajo creativo se paraliza; sin embargo, sigue cumpliendo con los compromisos no relacionados con la elaboración de obra original. El estado de tris- teza, abatimiento y desesperanza se convierte entonces en el objeto de consulta.

Aunque el paciente es bien consciente de ello por su formación en leyes, se le explica que toda la información proporcionada será tratada con la confidencialidad a la que obliga el secreto profesional.

\section{Estrategias de evaluación}

Como estrategias de evaluación se emplearon la entrevista clínica no estructurada, auto-registros y los siguientes autoinformes, habituales en la literatura en que la $\mathrm{AC}$ ha sido el tratamiento de elección:

Inventario de Depresión de Beck - II: El BDI-II (Beck, Steer y Brown, 1996) es la última versión del inventario de Beck. En este caso se empleó la versión en español baremada con muestras clínicas y no clínicas (Sanz, García-Vera, Espinosa, Fortín y Vázquez, 2003; Sanz, Perdigón y Vázquez, 2003).

Acceptance and Action Questionnaire: El AAQ (Hayes et al., 2004) es una medida del grado de evitación experiencial, conceptualizada a partir del modelo de la Terapia de Aceptación y Compromiso (Hayes, Strosahl y Wilson, 1999). La versión adaptada al español (Barraca, 2004) ha obtenido índices aceptables de consistencia interna (alfa de Cronbach $=, 74$ ), estabilidad temporal $\left(r_{\mathrm{XX}}=, 71\right)$ y ha reunido pruebas de $\mathrm{su}$ validez con muestras generales y clínicas.

Automatic Thought Questionnaire: El ATQ (Hollon y Kendall, 1980) es un cuestionario de 30 ítems desarrollado con el objetivo de medir la frecuencia de pensamientos automáticos negativos (frases negativas auto-dirigidas) frecuentemente asociados a la depresión. La adaptación española del ATQ aquí usada es la de Cano-García y RodríguezFranco (2002) que mantiene la estructura original de cuatro dimensiones (autoconcepto negativo, indefensión, ajuste pobre y autoreproches).

State-Trait Anxiety Inventory: El STAI-R / STAIE es una medida de la ansiedad, tanto en su vertiente de rasgo como de estado. En este caso se empleó la versión baremada en España (Spielberger, Gorsuch y Lushene, 1982).

Environmental Reward Observation Scale: El EROS (Armento y Hopko, 2007) es un breve instrumento de 10 ítems desarrollado para obtener una 
auto-evaluación objetiva del grado en que el entorno resulta reforzante para el sujeto. Una puntuación alta se vincula con un incremento de las conductas y del afecto positivo como consecuencia de experiencias reforzantes desde el medio. La escala posee una buena consistencia interna (alfa de Cronbach $=, 85$ ) $\mathrm{y}$ una buena fiabilidad test-retest $\left(r_{\mathrm{xx}}=, 85\right)$. También hay datos sobre su validez de constructo (correlaciones con el Pleasant Events Schedule y el BDI). La versión aquí usada es la adaptación al español realizada por Barraca y Pérez-Álvarez (2010).

Behavioral Activation for Depression Scale: El BADS (Kanter, Mulick, Busch, Berlin y Martell, 2007) es un cuestionario de 25 ítems elaborado para medir cuatro dimensiones básicas para el modelo de la AC: Activación, Evitación/Rumia, Afectación del trabajo/actividad y Afectación de la vida social. La versión usada en este caso es la adaptación al español realizada por Barraca y Pérez-Álvarez (pendiente de publicación).

Las puntuaciones del paciente en las escalas fueron las siguientes: BDI-II = 32 (depresión severa); $\mathrm{AAQ}=39$ (Evitación media-alta); ATQ total $=44$ (puntuación general alta); ATQ-I $=14$ (Autoconcepto Negativo alto); ATQ-II $=19$ (Indefensión alta); ATQ-III = 3 (Ajuste normal); ATQ-IV $=8$ (Autoreproches medio-alto); STAI-E $=41$ (ansiedad-estado alta); STAI-R = 39 (ansiedad-rasgo alta); EROS = 21 (reforzamiento desde el entorno bajo); BADS-I = 19 (activación baja); BADS-II = 19 (Evitación/Rumia alta); BADS-III = 15 (sin deterioro del trabajo o actividad); BADS-IV = 18 (deterioro de la vida social medio-alto $)^{1}$. Las mismas pruebas volvieron aplicarse tras la intervención, en la fase de seguimiento.

La entrevista clínica sirvió para conocer los aspectos más relevantes del caso y plantear el análisis funcional. Además, fue el método más importante para determinar el estado del paciente en el momento de acudir a consulta y también para valorar su evolución a lo largo de la terapia. Junto con el paciente se trató de establecer en cada sesión una

\footnotetext{
1 Excepto en el caso del BDI-II en el que las categorías están ya designadas, la puntuación obtenida se ha definido como alta cuando superaba una desviación típica la media y medio-alta cuando la puntuación típica $(Z)$ se encontraba entre 0,5 y 1 .
}

puntuación de 0 a 10 para determinar el estado de ánimo semanal. Aunque es posible que este tipo de estimaciones estén sesgadas por la subjetividad, aportan una información rápida y sencilla que resulta de gran utilidad para el clínico; además, son también de uso corriente en la evaluación del dolor crónico o de la ansiedad (como los conocidos "termómetros" para evaluar el nivel de miedo a determinados estímulos fóbicos).

Por último se pidió al paciente que elaborase auto-registros en los que reflejase las actividades diarias y el estado anímico asociado a cada una de ellas. No obstante, el paciente sólo cumplimentó esta tarea durante un único día. Tampoco se sirvió de diarios o correos electrónicos que, como alternativa a los registros, se le sugirieron.

\section{Formulación clínica del caso}

Las principales conductas problema aparecieron progresivamente, aunque en un breve intervalo de tiempo. Aproximadamente una semana después de la separación de su mujer, comenzaron a alterarse sus horarios, y el paciente empezó a acostarse y levantarse cada vez más tarde. Paralelamente, fue sufriendo un insomnio más acusado y el poco sueño que lograba se acompañaba de pesadillas. Se inició, así mismo, una disminución de peso, que llegó a suponer una pérdida de diez kilos, si bien es cierto que este proceso se explica en parte por la simultaneidad del problema con una gripe que duró cerca de dos semanas. Al tiempo, las autoafirmaciones del paciente se volvieron cada vez más negativas ("mi vida no tiene horizonte", "he perdido aquello que otorgaba sentido a todo mi esfuerzo: mi familia, mis hijos", "he fallado en aquello que más valoraba: comportarme con integridad", "vivo al límite de lo soportable").

En el plano cognitivo, se dieron otras manifestaciones problemáticas que acompañaron este proceso, como su recuerdo obsesivo de los momentos felices de su vida anterior, sentimientos de culpa y el planteamiento recurrente de que debía sufrir algún tipo de "tara", "deficiencia" que le habrían llevado a cometer la cadena de errores que explicaría su penosa situación actual. En esta misma línea, comentaba 
que su vida era últimamente pura apariencia y que desde bastantes años antes debía de arrastrar una serie de "problemas interiores", aunque nadie lo hubiese advertido.

En la esfera social también se observaron distintos problemas. Los contactos sociales generales eran evitados, pero se mantenían los familiares y aquellos a los que le obligaba su trabajo. No obstante, cuando estaba con sus padres disimulaba su verdadera situación anímica para evitarles sufrimiento.

La mayoría de estos problemas se intensificaba cada vez que hablaba con su ex pareja por los trámites del divorcio y los acuerdos del convenio. En las conversaciones surgían continuos reproches por temas económicos y por la educación de los hijos habidos en común. También exhibía un mayor decaimiento cuando su hija mayor (10 años) cuestionaba las razones de la separación.

Es necesario señalar en este caso que, aunque las verbalizaciones de malestar eran muy intensas (y, coherentemente, así lo reflejaban las puntuaciones de depresión del BDI-II o del ATQ), se producía la paradoja de que se mantenía una gran actividad incluso social - cuando el trabajo consistía en colaborar en medios de comunicación, impartir conferencias, ir a mesas en las que se hablaba de sus obras, etc. Igualmente, viajaba bastante, a veces conduciendo él mismo. El paciente mencionaba que se veía incapaz de soportar ese ritmo, aunque seguía haciéndolo y, a juzgar por los resultados, todas las tareas se efectuaban con gran eficacia. Entendía la contradicción entre lo que decía que sentía y lo que hacía, pero no sabía explicar por qué. No obstante, también es cierto que las actividades relativas a la creación de obra nueva estaban interrumpidas y que el paciente no se veía con capacidad para retomarlas; de hecho, las retrasaba indefinidamente.

Igualmente, vivía de forma ambivalente la posibilidad de recurrir a la medicación pues aunque, por un lado, se sentía enfermo, por otro temía depender de ella y, en general, cuando estaba más sereno, creía que sus problemas no tenían una naturaleza biológica.

Para explicar por qué el paciente se encontraba cada vez más deprimido a pesar de que seguía desarrollando una intensa actividad y siempre actuaba de forma responsable con sus obligaciones - algo en principio contradictorio con los procesos depresivos habituales - , se planteó un análisis funcional que justificaba la relación entre determinadas conductas y su estado anímico. Este análisis funcional se esbozó, en sus líneas generales, a partir del propuesto por los autores de la AC para dar cuenta del inicio y, fundamentalmente, del mantenimiento de la situación depresiva (Jacobson et al., 2001).

Desde este análisis se hipotetizó que, en un primer momento, el elemento disparador del estado depresivo se identificaba claramente con el proceso de ruptura matrimonial y las pérdidas asociadas a ésta (quebranto económico, disminución de la calidad de vida, empeoramiento del lugar de residencia, menor contacto con sus hijos, menor tiempo para sí mismo, etc.). Esta situación había desencadenado una serie de respuestas típicas de la condición depresiva (insomnio, pensamientos negativos - "nada tiene sentido" "cómo he podido cometer tantos errores"-, recuerdos intensos de la pasada vida feliz, dudas, pérdida de peso, culpabilidad, creerse enfermo, etc.). Sin embargo, lo más interesante fue observar que este tipo de respuestas se perpetuaba por el mantenimiento de una serie de patrones evitativos que estaban conformados por las variadas actividades que llevaba a cabo (viajes, conferencias, colaboraciones en medios de comunicación, etc.). Es decir, que, aunque el paciente se quejaba por verse obligado a llevar a cabo todas esas actividades, ello le justificaba para no sumergirse en una labor creativa como era redactar sus libros. Al mismo tiempo, su rumia negativa, su insomnio, su falta de fuerza, sus sentimientos de ineptitud y su recelo hacia las relaciones hombre-mujer, le empujaban a no concentrarse en la creación, le excusaban de pasar más tiempo con sus hijos y le tenían cerrado a iniciar cualquier otra relación de pareja. Por tanto - como predice el modelo de la $\mathrm{AC}$ - la situación depresiva provocaba determinados síntomas, pero igualmente podía verse como un comportamiento que mantenía la situación misma. Ciertamente, la publicación de un nuevo libro podía ser gratificante a largo plazo, pero a corto le obligaba a una importante labor de introspección, a trabajar solo y a ponerse en contacto con sus sentimientos. Del mismo modo, el volver a quedar con amigos y rehacer su red social e, incluso, volver a plantearse una nueva relación de pareja 
podía suponer un aliciente, pero en ese momento le recordaban lo que había perdido, especialmente su antigua estabilidad. En síntesis, la hipótesis que se planteó fue que la evitación que implicaba desarrollar muchas actividades laborales, junto con los mismos síntomas depresivos, favorecían un reforzamiento negativo inmediato (escapar de los sentimientos desagradables producidos por la ruptura), pero, lógicamente, cada vez le provocaban un mayor cansancio y le impedían a la larga acceder a reforzadores positivos más valiosos.

\section{Tratamiento}

\section{Elección del tratamiento}

Por lo revelado a partir del análisis funcional, se estimó que la AC constituía el tratamiento de elección. De los dos protocolos de intervención posibles, el de AC (Martell et al., 2001) y el del TACD (Lejuez et al., 2001), se eligió el primero de ellos por una serie de factores que parecían especialmente pertinentes en este caso. En concreto, el protocolo de AC: (1) enfatiza el papel del reforzamiento negativo en el mantenimiento de los estados depresivos; (2) se sirve de un conjunto más amplio de estrategias y no únicamente de la incorporación progresiva de tareas, circunstancia que ya se daba en este caso; (3) tiene en cuenta las manifestaciones depresivas cognitivas, como la rumia, también muy presente en este sujeto; y (4) existe evidencia empírica de su eficacia en casos semejantes en los que también había síntomas ansiosos y no se empleó medicación antidepresiva (Cullen, Spates, Pagoto y Doran, 2006; Santiago-Rivera et al., 2008).

\section{Aplicación del tratamiento}

A partir del análisis funcional se determinaron los objetivos del tratamiento y se inició su aplicación. Se consideraron los siguientes objetivos: (a) Conductas a activar: (1) Mantener unos horarios fijos (para acostarse, levantarse y trabajar en casa, desde el primer día de tratamiento empezaría ya fijando los de levantarse y acostarse), (2) Reiniciar la redacción de su último libro y mantener esta actividad (primero durante una hora al día como mínimo, hasta llegar a unas cinco horas) (3) Estar con sus hijos al menos un vez durante la semana y el fin de semana alterno que tiene asignado, (4) Volver a contactar con grupos de amigos y conocidos fines de semana alternos (cuando no le corresponda estar con sus hijos); (b) Conductas a extinguir o moderar: (1) Participar en la presentación de libros, conferencias, congresos, viajes, etc. sobre su obra (se acordó una disminución progresiva durante los siguientes meses que, al final, supusiera sólo una participación mensual), (2) Colaborar en medios de comunicación (se acordó que, tras cuatro meses, únicamente mantendría una colaboración estable con tres medios), (3) Desatender la rumia cognitiva (por medio de centrarse en la tarea presente - mindfulness - y valorar la función de esos pensamientos: ¿le activaban o facilitaban la pasividad y la evitación?).

Para llevar a cabo este plan de intervención, en primer lugar se explicó el análisis funcional y se justificó por qué unas conductas en principio positivas y activas (conferencias, tertulias, viajes, etc.) debían disminuirse progresivamente por suponer formas elaboradas de evitación; al tiempo, se justificó la importancia de acometer tareas difíciles cognitivamente - como la creación - , o delicadas y cansadas - como estar más con sus hijos y quedar con conocidos y amigos - , pero que, al final, podían volver a impregnar de sentido su vida y suponer un camino para salir de la presente situación. Hay que entender que la actividad creativa había configurado la vida de este paciente y el resto de su trabajo partía de esa vocación.

Tal y como plantea el modelo de AC, la consecución de estos objetivos tiene que establecerse de forma gradual, pues la situación depresiva es difícil de deshacer. Además, dado que no se puede esperar que el afrontamiento de las actividades señaladas produjesen rápidamente un alivio del estado depresivo sino todo lo contrario, era fundamental que se diese un compromiso por mantener esta actitud a lo largo de periodos más o menos extensos. Como es propio de la intervención de AC, las conductas a activar y a extinguir o moderar una vez incorporadas al tratamiento se mantuvieron de forma permanente.

La fase de aplicación de la terapia de AC en sí 
tuvo una duración de siete meses (a lo largo de 22 sesiones), tras un mes previo que sirvió como periodo de evaluación (5 sesiones), algo más largo de lo habitual por el detalle con que se recabaron las circunstancias vitales del sujeto y las dudas a la hora de establecer el análisis funcional. Justo después del fin del tratamiento, se programaron otras 5 sesiones de seguimiento cada vez más distanciadas en el tiempo (las dos primeras con un intervalo de quince días entre sí, luego las siguientes al cabo de veinte días, un mes y dos meses). En estas últimas se repitió la aplicación de autoinformes, se llevó a cabo una prevención de recaídas y se explicó la necesidad de seguir llevando a cabo una observación similar a la mostrada en consulta (análisis funcional) ante nuevas situaciones vitales potencialmente desencadenadoras de depresión (e.g. se enseñó a identificar patrones de evitación para responder con conductas de activación). El cronograma de las sesiones puede verse en la tabla 1. Tal y como plantea la AC, la incorporación de las estrategias y de los objetivos señalados fue progresiva, y de este modo aparece recogido en la tabla.

Durante el proceso de implementación de la terapia se produjeron algunos parones, motivados tanto por los periodos vacacionales como por la necesidad de atender a sus obligaciones laborales fuera del lugar de residencia. Aunque al principio fue dubita- tivo, el compromiso con las tareas y la corrección de algunas estrategias facilitó la inclusión de los siguientes elementos. Como el estado anímico y la confianza del paciente se asentaron al cabo de varios meses, las últimas incorporaciones de conductas a extinguir (por ejemplo, desatender el contenido de la rumia depresiva y centrarse en la experiencia directa) resultó más sencilla y presentó menos reservas por parte del paciente.

\section{Factores relacionados con el terapeuta}

Las sesiones fueron conducidas por un único terapeuta, autor de este artículo. El terapeuta finalizó su licenciatura en 1992, realizó un Máster en Psicología Clínica y de la Salud entre 1992-1994 y se doctoró en 1997. Está en posesión del Título de Psicólogo Especialista en Psicología Clínica y de Psicólogo Especialista en Psicoterapia (EFPA). Las sesiones se llevaron a cabo en la consulta privada del terapeuta.

La relación terapéutica podría considerarse buena. El paciente mostró adherencia al tratamiento y trató de cumplir con las prescripciones. No obstante, al inicio de la intervención no completó los registros recomendados. Es importante señalar que el paciente había tenido experiencias negativas con

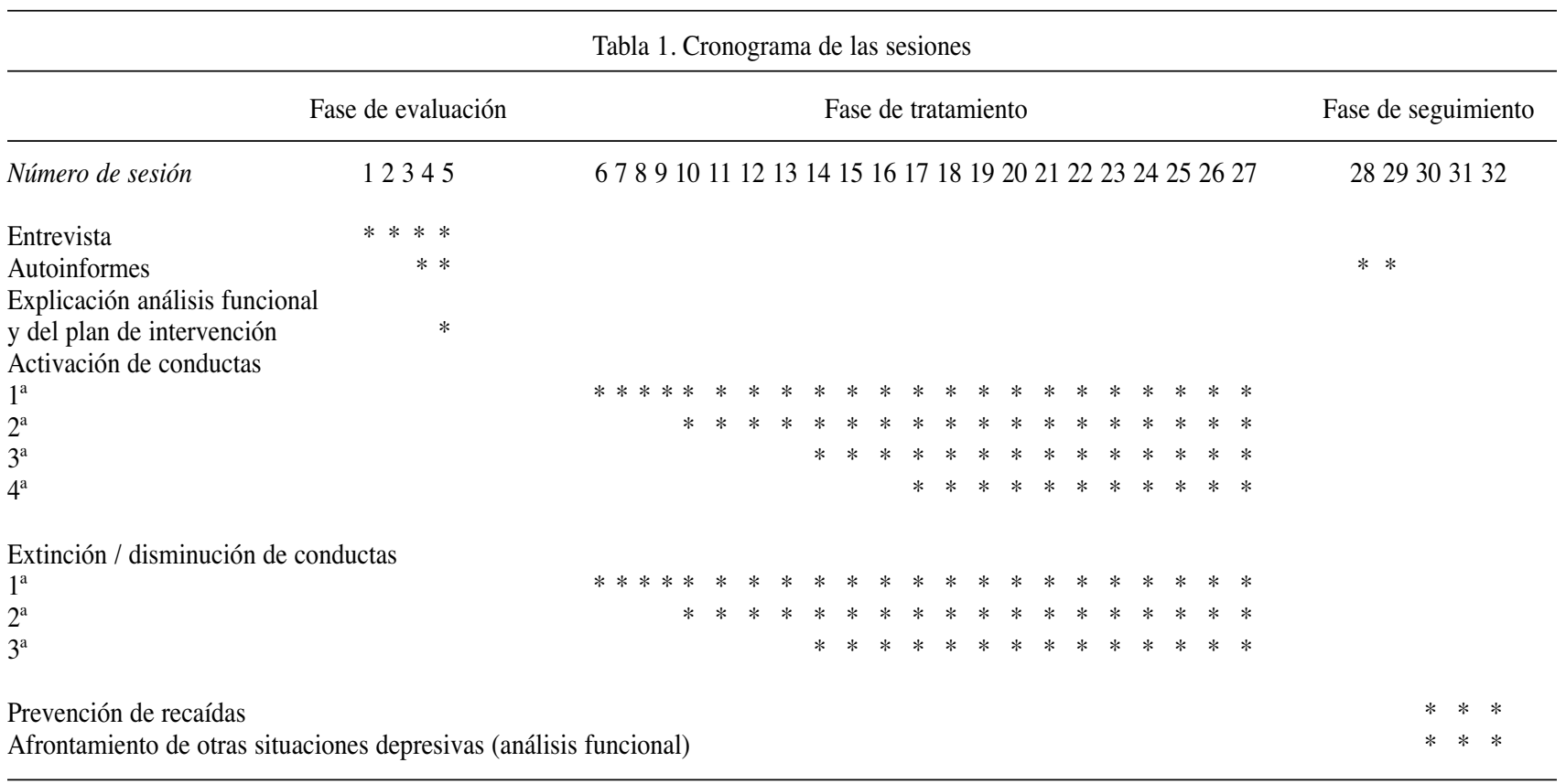


psiquiatras y que esta circunstancia podía afectar a su confianza inicial hacia cualquier proceso terapéutico. Más adelante, el paciente regaló libros propios al terapeuta y estuvo dispuesto a acudir con más frecuencia cuando se le indicó la pertinencia por encontrarse peor anímicamente y por dar la adecuada continuidad al tratamiento. El terapeuta mostró interés por los libros regalados y los comentó en sesión. Las actividades culturales del paciente eran genuinamente interesantes para el terapeuta, lo que probablemente se reflejó en el curso de la terapia. El paciente no canceló las citas programadas y siempre avisó con tiempo si se veía obligado a cambiarlas. Acudió también a las sesiones de seguimiento pese a estar distanciadas en el tiempo.

\section{Diseño del estudio}

El caso aquí reportado se corresponde con un diseño AB. Si bien por este tipo de diseño no es posible determinar con completa seguridad que los cambios obedeciesen únicamente a la aplicación de la terapia de $\mathrm{AC}$, puede mencionarse que durante la intervención no se dieron cambios familiares, laborales o contextuales importantes, por lo que existe una mayor confianza en que fuesen las estrategias de la terapia las que explican las mejoras observadas.

\section{Análisis de datos}

A fin de obtener medidas para contrastar el posible cambio por la intervención, en el seguimiento se repitió la administración de todos los autoinformes aplicados durante la línea base. Por otro lado, puesto que el paciente no completó los auto-registros que se prescribieron al inicio de la intervención, fue necesario recurrir a otras estrategias para valorar la efectividad de la terapia. En concreto, el terapeuta estimó, junto con el paciente, el estado de ánimo durante la semana (con un rango de puntuaciones de 0 a 10). Además, se registró el cumplimiento de las tareas asignadas como objetivos de la intervención (conductas a activar y conductas a extinguir o moderar).

Teniendo en cuenta la naturaleza de estas medidas, se ha considerado que la presentación de los resultados en formato visual resultaría más clara. La figura 1 recoge las diferencias entre las medidas pre y postratamiento en cada uno de los autoinformes aplicados. La figura 2 presenta integrados los datos de la escala de apreciación del estado anímico

Figura 1. Puntuaciones pre y postratamiento en los autoinformes empleados

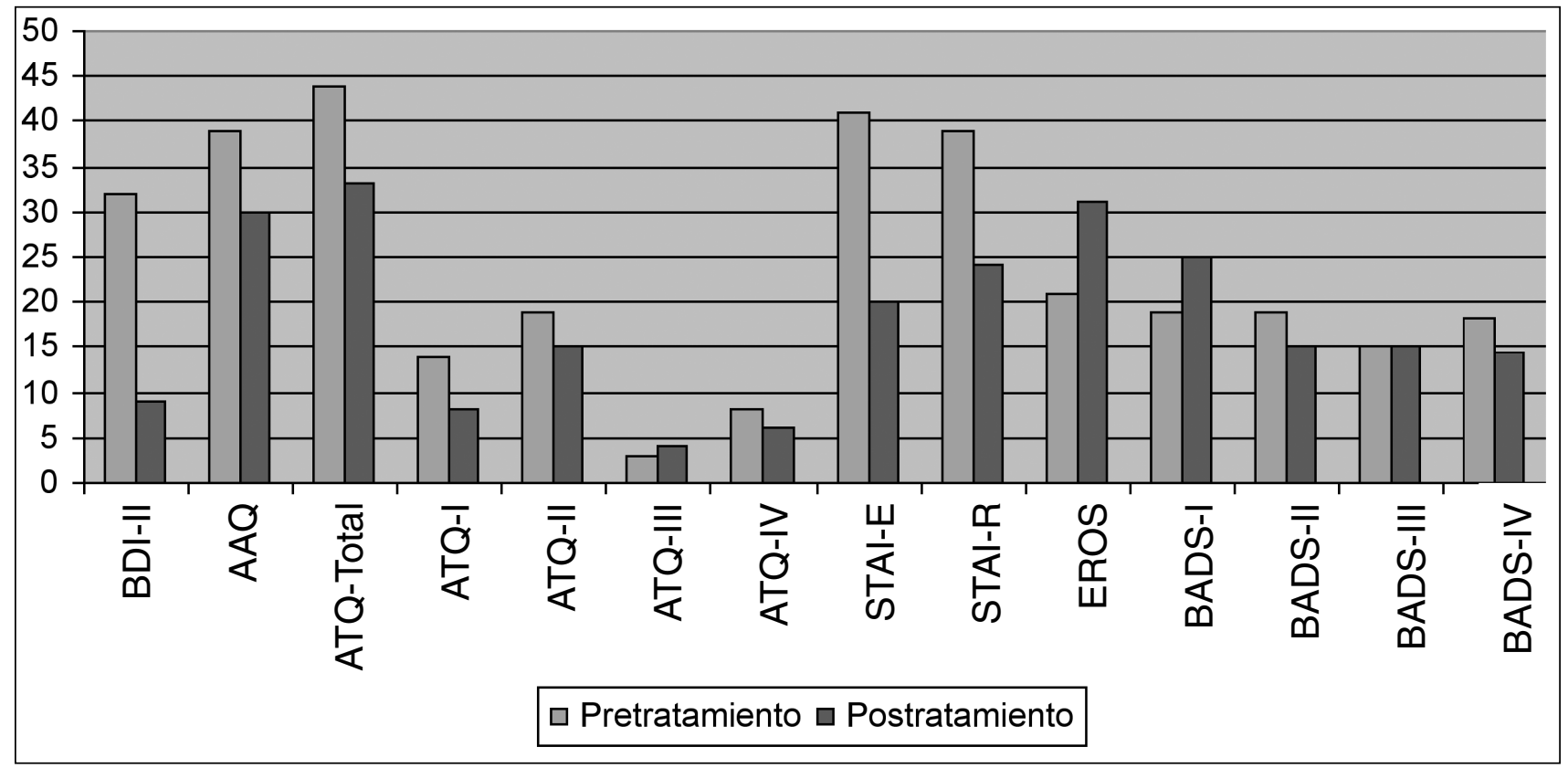


durante toda la terapia y en el seguimiento (cinco meses después) junto con el cumplimiento (en número) de las tareas a activar, a disminuir y extinguir, que se recabaron semana a semana en cada sesión con la información proporcionada por el paciente.

\section{Efectividad y eficiencia de la intervención}

Los resultados de la intervención pueden comprobarse al observar las notables diferencias entre la mayoría de las puntuaciones de los autoinformes previas y posteriores a la fase de intervención (ver figura 1). En el BDI-II se pasó de una puntuación de 32 (depresión severa) a una de 9 (ausencia de depresión). En el AAQ el cambio se produjo desde una puntuación de 39 a una de 30. En este caso, hay que tener en cuenta que en la adaptación española del instrumento la puntuación media en la población general (no clínica) es de 34,61 (DT $=5,43$ ) (Barraca, 2004). En el ATQ la puntuación general pasó de 44 a 33, y en las cuatro dimensiones: (1) Autoconcepto Negativo de 14 a 8; (2) Indefensión de 19 a 15; (3) Pobre ajuste de 3 a 4; y (4) Autoreproches de 8 a 6 . En el STAI hubo un cambio importante en las dos dimensiones del instrumento, pasando de 41 a 20 en la ansiedad estado, y de 39 a 24 en la ansiedad rasgo, lo que supone pasar del Pn 95 al 55 en el primer caso y del Pn 97 al 70 en el segundo en el baremo de los hombres adultos (Spielberger et al., 1982). En el EROS se produjo otro cambio significativo desde los 21 puntos iniciales hasta 31. En la prueba original la media para la población general masculina es de 29.61 (DT = 4.20) (Armento y Hopko, 2007). Por último, en la primera dimensión del BADS (Activación) el paciente pasó de 19 puntos a 25, en la segunda dimensión (Evitación/Rumia) de 19 a 15, en la tercera (Deterioro del trabajo) no se produjeron cambios (de 15 a 15) y en la cuarta (Deterioro de la vida social) de 18 a 14. En este caso, los valores medios reportados por Barraca y Pérez-Álvarez (pendiente de publicación) para cada subescala son 21.36 (DT $=8.93), 14.55(\mathrm{DT}=6.67), 16.37(\mathrm{DT}=8.09) \mathrm{y}$ $14.87(\mathrm{DT}=10.17)$.

La figura 2 revela los notables cambios en el estado anímico y el cumplimiento tanto de la activación de las conductas objetivo como de la extinción de los patrones de evitación detectados. Aunque es difícil determinar la magnitud de estos cambios, en particular al inicio del tratamiento, pues la intervención tuvo que empezar sin que se hubiese alcanzado la deseable estabilidad en la línea base, la apreciación

Figura 2. Gráfico de la evolución del estado de ánimo, la activación de conductas y la extinción de conductas a lo largo de la intervención (las líneas discontinuas separan las fases de evaluación, intervención y seguimiento)

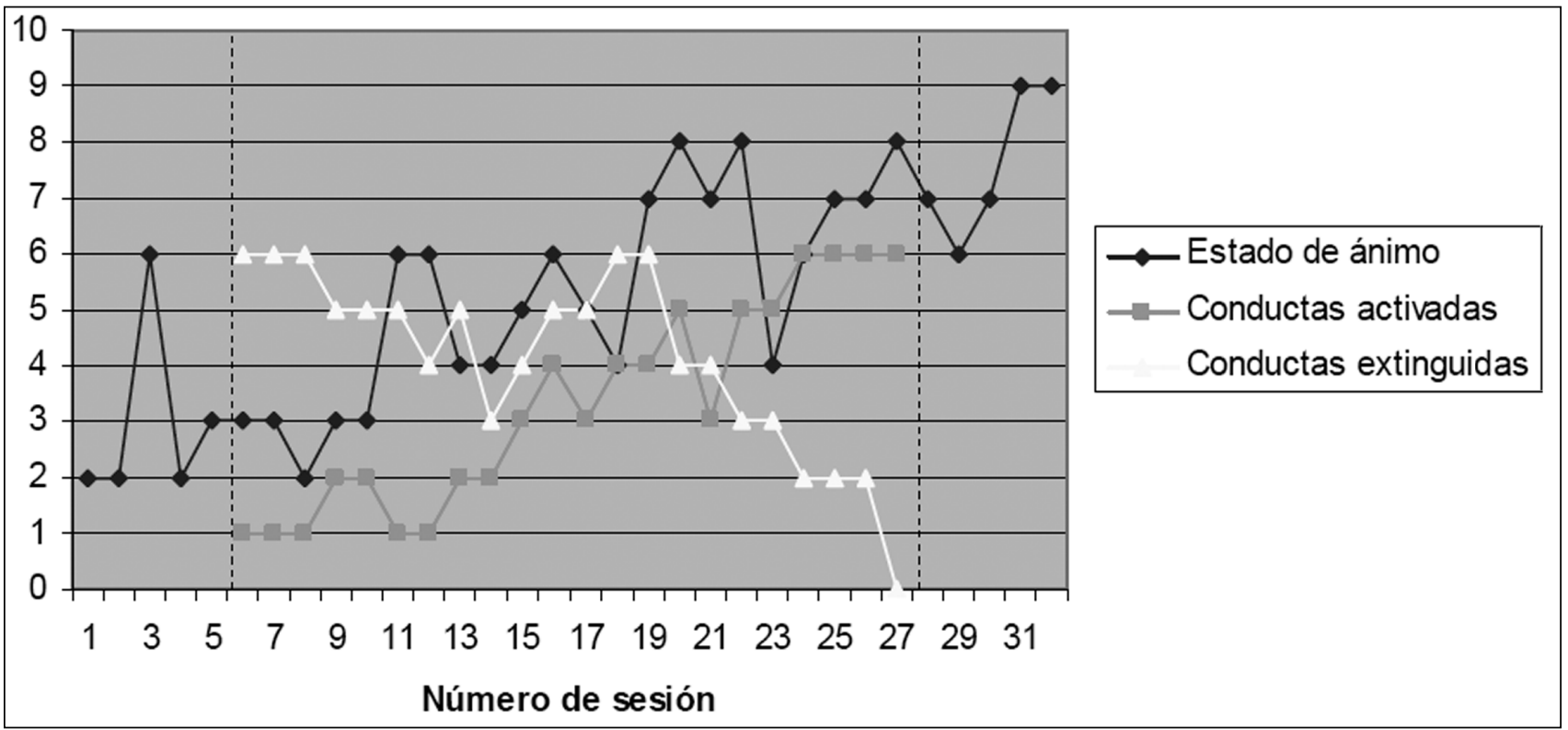


visual del gráfico invita a suponer unas mejoras significativas como consecuencia de la intervención. Esta relación se corresponde con la planteada por el modelo de AC, que esgrime que la desaparición progresiva de las conductas de evitación y el cese del reforzamiento de las conductas depresivas conduce, a la larga, a una mejora del estado anímico.

Junto con estas medidas, puede señalarse que el paciente expresó su satisfacción por la terapia e informó encontrarse plenamente recuperado. Manifestó que veía claro que había llegado el momento del alta y estuvo dispuesto a ofrecer información posterior si se le requería para seguimientos a más largo plazo, como de hecho fue el caso. Dado que nunca había dejado de trabajar ni de relacionarse con otras personas, estos comentarios son un importante criterio de su mejoría.

El método de intervención de la $\mathrm{AC}$, en el que se enfatiza la importancia de asimilar el análisis funcional y, por tanto, de comprender la relación entre la realización de determinadas actividades y el estado de ánimo (Dimidjian et al., 2008), supone una garantía de que la mejoría anímica se mantendrá a lo largo del tiempo, y así parecen indicarlo también los datos de este caso en el seguimiento, en el que, incluso, el estado de ánimo al cabo de cinco meses era mejor que justo al final de la fase de tratamiento.

\section{Discusión}

Las mejorías observadas en este paciente tras la intervención representan un aval importante para el modelo planteado por la terapia de AC en el caso de los trastornos del estado de ánimo. No obstante, es importante señalar que la elección de esta terapia no partió tanto de la sintomatología depresiva cuanto del análisis funcional elaborado. En concreto, la insistencia de la AC por apreciar la funcionalidad de la conducta y no establecer apriorismos sobre cómo son los comportamientos de evitación fue clave para la comprensión de la situación. Aparentemente, el paciente llevaba una vida muy activa, pero su "situación depresiva" era muy intensa porque toda su laboriosidad estaba al servicio de la evitación de acciones comprometidas y le excusaban de enfren- tarse a tareas realmente difíciles anímicamente. Por eso, un tratamiento convencional en el que se pautasen sólo actividades diarias gratificantes que incorporar de forma progresiva, como el propuesto por Lewinsohn y sus colaboradores (Lewinsohn y Graf, 1973; Lewinsohn, Muñoz, Youngren y Zeiss, 1978; Zeiss, Lewinsohn y Muñoz, 1979), habría resultado de dudosa efectividad.

Por otro lado, es posible que una terapia de corte cognitivo se hubiese centrado en la necesidad de modificar las creencias (por ejemplo, las relativas a estar enfermo, tener algún tipo de tara o deficiencia, o haber perdido el sentido de su vida) dada su falta de evidencia empírica; sin embargo, esto no hubiese facilitado la comprensión de que estos pensamientos - esa "rumia depresiva" - podía estar al servicio de la evitación de otras actividades asociadas a las pérdidas y cambios familiares. La AC no deja de tener como objetivo que desaparezcan las cogniciones distorsionadas del paciente, pero espera que esos cambios se produzcan como consecuencia de la activación misma (Kanter et al., 2007; Lejuez et al., 2001; Martell et al., 2001) y, cuando se abordan en sesión, es para considerar no la veracidad de sus contenidos, sino su función: ¿sirven para encaminarse o para alejarse de los objetivos planteados?, ¿cuánto tiempo roban?, ¿qué otras cosas podrían hacerse en vez de rumiar? (Martell et al., 2001). De hecho, en este sujeto, el descenso de la puntuación en la segunda dimensión del BADS (Evitación/ Rumia) es un dato a favor del cambio cognitivo como producto indirecto de la activación.

El caso aquí presentado ha procurado ilustrar el modelo propuesto por la $\mathrm{AC}$ y la intervención que de él se deriva en un paciente probablemente poco convencional respecto a su proceso depresivo, pero, quizás precisamente por ello, más útil para entender lo que esta terapia ofrece como novedoso. Como se ha podido comprobar, lo importante no es tanto la aplicación de un protocolo con un apoyo experimental bien justificado, sino subrayar el valor que el análisis funcional puede tener en las ocasiones en que existe la posibilidad de disponer de más sesiones para una aproximación individualizada (como es el caso de la consulta privada). No obstante, es cierto que para otro tipo de contextos, como los institucionales, el protocolo más parsimonioso pro- 
puesto por la TACD resulta una elección más justificable.

El presente caso clínico posee una serie de limitaciones que es necesario señalar. Para empezar, es cierto que la falta de auto-registros ha dificultado ofrecer una valoración del caso complementaria. Además - como han planteado los mismos autores de la $\mathrm{AC}$ - estos registros son un medio importante para facilitar la comprensión del paciente del análisis funcional $\mathrm{y}$, por tanto, un elemento valioso para la prevención de las recaídas. No obstante, también es verdad que los auto-registros son muchas veces poco motivantes para pacientes aquejados de una depresión severa y con experiencias previas negativas sobre su cumplimentación. En este caso su ausencia no impidió llevar a cabo la intervención con éxito. Por otro lado, su falta no debería bloquear su divulgación en la literatura científica. Junto con esta limitación, podría dejarse apuntada la escasez de estudios de algunos de los autoinformes empleados, en particular del EROS y del BADS, pues si bien éstos han mostrado ya unas propiedades psicométricas adecuadas, carecen aún de estudios de validación en la población española aparte de los mencionados en este mismo artículo. En cualquier caso, se estimó útil incluirlos en la evaluación del paciente por estar diseñados específicamente para el trata- miento de la AC. Por último, cabe dudar del efecto exclusivo de la intervención como fuente de la mejoría del paciente al tratarse de un diseño $A B$ en un entorno poco controlado (consulta privada externa). Se debe ser consciente de que determinados cambios, en particular los relativos a los niveles de ansiedad, pueden explicarse en ocasiones por un proceso de habituación a la situación terapéutica, entre otras posibles razones.

La AC resulta una terapia muy prometedora. Sus principios son bien comprendidos por los terapeutas cognitivo-conductuales y sus métodos resultan más familiares que los de otras terapias de la tercera generación, como la Terapia de Aceptación y Compromiso, la Psicoterapia Analítico Funcional o la Terapia Dialéctica Comportamental. En su breve vida, la AC ha reunido un considerable aval experimental en estudios altamente controlados. Con todo, resulta imprescindible que siga divulgándose y que se prodiguen los estudios de caso que favorezcan el deseo de incorporarla al quehacer de los psicólogos clínicos. Idealmente, la investigación futura de casos clínicos con la AC debe tratar de incorporar otros diseños más complejos (por ejemplo, $\mathrm{ABAB}$, $\mathrm{ABCB}$, línea base múltiple, etc.) que permitan una mayor seguridad en la interpretación de los cambios producidos por la terapia.

\section{Extended Summary}

The article describes in detail intervention in a subject with symptoms of depression. After acquiring the basic information required for functional analysis, the pertinence of using Behavioral Activation Therapy (BA) became clear. Its good results and the simplicity of its techniques, in spite of the severity of the symptoms, justify publicizing it.

BA is a behavioral treatment developed for depression. Although its focus goes back to the work of Ferster (1973) and Lewinsohn (1974), as well as studies by Jacobson on disassembling effective cognitive therapy components (Gortner, Gollan, Dobson, \& Jacobson, 1998; Jacobson et al., 1996), its current body is also the fruit of other influences (for example, Beck's behavioral techniques or the contributions of third-generation behavioral therapies), so its systematization has only really come about in the last decade. Today, BA may be considered a therapy in its own right with an original explanatory framework, innovative evaluation procedures and renovated intervention techniques.

The current BA model (Martell, Addis, \& Jacobson, 2001; Martell, Dimidjian, \& HermanDunn, 2010) considers depression to be a logical response to the situation in which a person is immersed, maintaining avoidance behavior to escape from discomfort, pain or psychological distress. It sets out rewards that would lead in the long term to a better adjusted and more gratifying existence, which had previously been impossible. Although the start of the manifestations of depression is usually found in a significant change or loss (which may occur suddenly or gradually), the process that maintains the state of depression is located in the interaction which the subject estab- 
lishes with the environment from a certain time onward. Depression is thus conceived as a whole situation in which one is immersed, and not only as a dysphoric emotional state. Its characteristic symptoms (for example, negative ideation, anhedonia, loss of energy, attention and concentration, social isolation, abandonment, changes in eating or sleeping, etc.) are conceived as behaviors that perpetuate this situation. Logically, from this point of view, cognitive deficit or neurochemicals are not relevant, nor does the term "disease" make sense, but rather the exact opposite. "The sense" that behaviors have is emphasized to find relief (as the means of escape that they are), although in the long term they become unadapted and provoke more and more suffering.

Today, BA has acquired broad evidence for its efficacy, both in well-controlled experimental works (Dimidjian et al., 2006; Gawrysiak, Nicholas, \& Hopko, 2009), and in case studies (Bottonari, Roberts, Thomas, \& Read, 2008; Hopko, Lejuez, \& Hopko, 2004; Hopko, Robertson, \& Lejuez, 2006; Santiago-Rivera, Kanter, Benson, Derose, Illes, \& Reyes, 2007), such as the one described in this article.

The subject treated was a 44-year-old male with severe symptoms of depression and anxiety. He came for consultation a few weeks after having separated from his wife. This separation was motivated by the patient's infidelity, and took place in a very tense atmosphere. The case was evaluated by means of several self-reports (BDI-II, ATQ, AAQ, STAI, EROS and BADS), by an unstructured clinical interview and self-records (although these were only partly answered). The evaluation took five weeks. The most notable symptoms in the beginning were profound sadness, despondency and desperation, and, associated with them, a wide set of cognitive, social and personal problems among which were marked alteration of sleep (in particular, difficulty falling asleep and doing so late, nightmares and unrefreshing sleep), appetite (the subject lost ten kilograms), negative ideation and self-guilt (ideas about death, of leading a life without sense, of having failed in everything important, of having some mental defect or damage, etc.) and of being incapable of maintaining his work commitments and the activity which he was carrying out at that time.
The information acquired established the basal mood state, as well as the type and level of his activity and also abandoned activities. Through a detailed examination of his behavioral dynamics and of associated feelings, a functional analysis was formulated which revealed how many of the activities he carried out had an escape/avoidance function. The BA model turned out to be coherent in explaining why in reality the subject was so depressed, although his work was intense and varied (lectures, courses, permanent collaboration with media, etc.), which in principle should have translated into a higher mood state. In fact, all the patients work was at the service of avoidance, since that way he was not in contact with the reality of his family loss, did not confront other tasks which were more important to him according to his values, and the sense that he had wanted to imprint on his life (that is, be a writer and write a new book) and did not relate with friends or any possible future partner. The model also made sense of his main symptoms, since the sleep affection, which wrecked his schedules, his bad health, ruminating negative thoughts and the vivid memory of his past happy life now lost, "impeded" him from making vital changes, so they became maintainers of his current state.

Treatment of the case, which took seven months (in 22 sessions), began with a detailed explanation of the intervention model and the functional analysis, which justified the process to be followed. According to the logic described, we decided to activate certain behavior connected to the patient's values and likely to provide long-term positive reinforcement, as well as extinguish others, which, although reinforcing in the short term, were no more than forms of avoidance. Thus, more specifically, we agreed on progressive activation of: (1) Keeping fixed schedules (for going to bed, getting up and working); (2) Starting to write his latest book again and keeping this activity up (first for an hour a day at least, and later up to five hours); (3) Being with his children at least once a week and alternating weekends assigned to him; and (4) Getting in touch with groups of friends and acquaintances on alternating weekends (when he was not supposed to be with his children). And extinction or progressive moderation of the following behavior: (1) Participating in 
fewer presentations, lectures, congresses, trips, etc., for his work (we decided on progressive reduction during the following months until at the end it was down to one a month); (2) collaborating with fewer media (we agreed that, after four months, he would only keep up stable collaboration with three of the media); and (3) not paying attention to his cognitive rumination (by concentrating on the task at hand and assessing the function of those thoughts: were they activating him or facilitating passivity and avoidance?). Finally, the complete set of strategies proposed for treatment was made up of monitoring his mood state, positive reinforcement, extinction, mindfulness and self-motivation toward action.

The results of the intervention were found from the pos-test measurements by questionnaires, evolution on a subjective scale of well-being (collected throughout the intervention) and analysis of the number and type of activities carried out. After Session 22 of the intervention, the symptoms of depression had decreased noticeably and qualitative differences were found in the scores on the questionnaires - for example, on the BDI-II, he went from a score of 32 (severe depression) to a score of 9 (absence of depression); on the ATQ the general score went from 44 to 33 and on the four dimensions, (1) Negative self concept from 14 to 8; (2) Hopelessness from 19 to 15; (3) Poor adjustment from 3 to 4; and (4) Self reproach from 8 to 6; on the STAI there was a change in anxiety state from 41 to 20 points, which means going from Pn 95 to 55. On the subjective scale of well-being, he went from a score of 2 to 8 . Finally, it was found that the patient had applied himself to incorporating activation behavior and eliminating or moderating behavior identified with avoidance. Follow-up showed that the benefits provided by the intervention were still maintained five months later, both in terms of no reappearance of depression symptoms and subjective manifestation of well-being.

All together, the results of this intervention join the already extensive list of publications showing the usefulness of BA, naturally with the limitations inherent to single cases. It is important to emphasize the value that functional analysis had in this case. The functional analysis was an idiographic treatment which did not include more supposedly- gratifying activities which again might be in the service of avoidance, made sense, as did conceiving distorted thoughts not as the origin of the patient's depressive discomfort (and therefore, focus of the therapeutic action), but just one more avoidance behavior and, therefore, tending to perpetuate the situation. One important problem of the intervention was the lack of record keeping by the patient, which lengthened evaluation and endangered monitoring of the mood state and comprehension of the model, fundamental aspects according to the authors of BA. Nevertheless, judging by the results, these drawbacks were not insurmountable obstacles.

\section{Referencias}

Armento, M. E. A. y Hopko, D. R. (2007). The Environmental Reward Observation Scale (EROS): Development, Validity, and Reliability. Behavior Therapy, 38, 107-119.

Barraca, J. (2004). Spanish Adaptation of the Activation and Action Questionnaire (AAQ). International Journal of Psychology and Psychological Therapy, 4, 505-515.

Barraca, J. (2006). Editorial. Las terapias de conducta de tercera generación: ¿parientes políticos o hermanos carnales? EduPsykhé, 5, 147-157.

Barraca, J. (2009). La Activación Conductual (AC) y la Terapia de Activación Conductual para la Depresión (TACD): Dos protocolos de tratamiento desde el modelo de la activación conductual. EduPsykhé, 8, 23-47.

Barraca, J. y Pérez-Álvarez, M. (2010). Adaptación española del Environmental Reward Observation Scale (EROS). Ansiedad y Estrés, 16, 95-107.

Barraca, J. y Pérez-Álvarez, M. (pendiente de publicación). Avoidance and Activation as Keys to Depression: Adaptation of the Behavioral Activation for Depression Scale (BADS) in a Spanish Sample.

Beck, A. T., Rush, A. J., Shaw, B. F. y Emery, G. (1979). Cognitive therapy of depression. Nueva York: Guilford Press.

Beck, A. T., Steer, R. A. y Brown, G. K. (1996). Manual for the BDI-II. San Antonio, TX: Psychological Corporation. 
Bottonari, K.A., Roberts, J. E., Thomas, S. N. y Read, J. P. (2008). Stop thinking and start doing: Switching from cognitive therapy to behavioral activation in a case of chronic treatment-resistant depression. Cognitive and Behavioral Practice, 15, 376-386.

Cano-García, F. J. y Rodríguez-Franco, L. (2002). Evaluación del lenguaje interno ansiógeno y depresógeno en la experiencia de dolor crónico. Apuntes de Psicología, 20, 329-346.

Cuijpers, P., van Straten, A. y Warmerdam, L. (2007). Behavioral activation treatments of depression: A meta-analysis. Clinical Psychology Review, 27, 318-326.

Cullen, J. M., Spates, C. R., Pagoto, S. y Doran, N. (2006). Behavioral Activation Treatment for Major Depressive Disorder: A Pilot Investigation. The Behavior Analyst Today, 7, 151-166.

Dimidjian, S., Hollon, S. D., Dobson, K. S., Schmaling, K. B., Kohlenberg, R. J., Addis, M. E., et al. (2006). Randomized Trial of Behavioral Activation, Cognitive Therapy, and Antidepressant Medication in the Acute Treatment of Adults with Major Depression. Journal of Consulting and Clinical Psychology, 74, 658-670.

Dimidjian, S., Martell, C. R., Addis, M. E. y Herman-Dunn, R. (2008). Behavioral Activation for Depression. En D. H. Barlow (Ed.), Clinical Handbook of Psychological Disorders. A step-bystep treatment manual (4 ${ }^{a}$ ed.) (pp. 328-364). Nueva York: Guilford Press.

Dobson, K. S., Hollon, S. D., Dimidjian, S., Schmaling, K. B., Kohlenberg, R. J., Gallop, R., et al. (2008). Randomized Trial of Behavioral Activation, Cognitive Therapy, and Antidepressant Medication in the Prevention Relapse and Recurrence in Major Depression. Journal of Consulting and Clinical Psychology, 76, 468-477.

Ferster, C. B. (1973). A functional analysis of depression. American Psychologist, 28, 850-870.

Gawrysiak, M., Nicholas, C. y Hopko, D. R. (2009). Behavioral activation for moderately depressed university students: Randomized controlled trial. Journal of Counseling Psychology, 56, 468-475.

Gortner, E. T., Gollan, J. K., Dobson, K. S. y
Jacobson, N. S. (1998). Cognitive-behavioral treatment for depression: Relapse prevention. Journal of Consulting and Clinical Psychology, 66, 377-384.

Hayes, S. C. (2004). Acceptance and Commitment Therapy, Relational Frame Theory, and Third Wave of Behavioral and Cognitive Therapies. Behavior Therapy, 35, 639-665.

Hayes, S. C., Strosahl, K. D. y Wilson, K. G. (1999). Acceptance and Commitment Therapy. An Experiential Approach to Behavior Change. Nueva York: Guilford Press.

Hayes, S. C., Strosahl, K. D., Wilson, K. G., Bissett, R. T., Pistorello, J., Toarmino, D., et al. (2004). Measuring experiential avoidance: A preliminary test of a working model. The Psychological Record, 54, 553-578.

Hollon, S. D. y Kendall, P. C. (1980). Cognitive self-statements in depression: Development of an automatic thoughts questionnaire. Cognitive Therapy and Research, 4, 383-395.

Hopko, D. R., Lejuez, C. W. y Hopko, S. D. (2004). Behavioral activation as an intervention for coexistent depressive and anxiety symptoms. Clinical Case Studies, 3, 37-48.

Hopko, D. R., Lejuez, C. W., LePage, J., Hopko, S. D. y McNeil, D. W. (2003). A brief behavioral activation treatment for depression: A randomized trial within an inpatient psychiatric hospital. Behavior Modification, 27, 458-469.

Hopko, D. R., Lejuez, C. W., Ruggiero, K. J. y Eifert, G. H. (2003). Contemporary behavioral activation treatment for depression: Procedures, principles, and progress. Clinical Psychology Review, 23, 699-717.

Hopko, D. R., Robertson, S. M. C. y Lejuez, C. W. (2006). Behavioral Activation for Anxiety Disorders. The Behavior Analyst Today, 7, 212232.

Jacobson, N. S., Dobson, K. S. Truax, P. A., Addis, M. E., Koerner, K., Gollan, J. K., et al. (1996). A component analysis of cognitive-behavioral treatment for depression. Journal of Consulting and Clinical Psychology, 64, 295-304.

Jacobson, N. S. y Gortner, E. T. (2000). Can depression be demedicalized in the 21st century: Scientific revolutions, counter-revolutions, and 
the magnetic field of normal science. Behaviour Research and Therapy, 38, 103-117.

Jacobson, N. S., Martell, C. R. y Dimidjian, S. (2001). Behavioral activation treatment for depression: Returning to contextual roots. Clinical Psychology: Science and Practice, 8, 255-270.

Kanter, J. W., Busch, A. M. y Rusch, L. C. (2009). Behavioral Activation: Distinctive Features. Nueva York: Routledge.

Kanter, J. W., Mulick, P. S., Busch, A. M., Berlin, K. S. y Martell, C. R. (2007). The Behavioral Activation for Depression Scale (BADS): Psychometric Properties and Factor Structure. Journal of Psychopathology and Behavior Assessment, 29, 191-202.

Lejuez, C. W., Hopko, D. R. y Hopko, S. D. (2001). A brief Behavioral Activation Treatment for Depression: Treatment Manual. Behavior Modification, 25, 225-286.

Lejuez, C. W., Hopko, D. R. y Hopko, S. D. (2002). The brief Behavioral Activation Treatment for Depression (BATD): A comprehensive patient guide. Boston, MA: Pearson Custom Publishing. Lejuez, C. W., Hopko, D. R., LePage, J., Hopko, S. D. y McNeil, D. W. (2001). A brief Behavioral Activation Treatment for Depression. Cognitive and Behavioral Practice, 8, 164-175.

Lewinsohn, P. M. (1974). A behavioral approach to depression. En R. M. Friedman y M. M. Katz (Eds.), The psychology of depression: Contemporary theory and research (pp. 157185). Nueva York: John Wiley.

Lewinsohn, P. M. y Graf, M. (1973). Pleasent activities and depression. Journal of Consulting and Clinical Psychology, 41, 261-268.

Lewinsohn, P. M., Muñoz, R. F., Youngren, M. A. y Zeiss, A. M. (1978). Control your depression. Englewoods Cliffs, NJ: Prentice Hall.

Martell, C. R., Addis, M. E. y Jacobson, N. S. (2001). Depression in context: Strategies for guided action. Nueva York: W. W. Norton.

Martell, C. R., Dimidjian, S. y Herman-Dunn, R.
(2010). Behavioral Activation for Depression: A Clinician's Guide. Nueva York: Guilford Press.

Pagoto, S., Bondenlos, J. S., Schneider, K. L., Olendzki, B., Spates, C. R. y Ma, Y. (2008). Initial investigation of behavioral activation therapy for co-morbid major depressive disorder and obesity. Psychotherapy: Theory, Research, Practice, Training, 45, 410-415.

Pérez-Álvarez, M. (2006). La terapia de conducta de tercera generación. EduPsykhé, 5, 159-172.

Pérez-Álvarez, M. (2007). La Activación Conductual y la desmedicalización de la depresión. Papeles del Psicólogo, 28, 97-110.

Santiago-Rivera, A., Kanter, J., Benson, G. Derose, T., Illes, R. y Reyes, W. (2008). Behavioral Activation as an alternative treatment approach for latinos with depression. Psychotherapy: Theory, Research, Practice, Training, 45, 173185.

Sanz, J., García-Vera, M. P., Espinosa, R., Fortín, M. y Vázquez C. (2003). Adaptación española del Inventario para la Depresión de Beck-II (BDI-II):

3. Propiedades psicométricas en pacientes con trastornos psicológicos. Clínica y Salud,16, 121142.

Sanz, J., Perdigón, A. L. y Vázquez C. (2003). Adaptación española del Inventario para la Depresión de Beck-II (BDI-II): 2. Propiedades psicométricas en población general. Clínica y Salud, 14, 249-280.

Spielberger, C. D., Gorsuch, R.L. y Lushene, R.E. (1982). STAI. Cuestionario de Ansiedad Estado/Rasgo. Madrid: TEA, Ediciones.

Virués-Ortega, J. y Moreno-Rodríguez, R. (2008). Guía para la realización de informes de casos clínicos en psicología clínica conductual. International Journal of Clinical and Health Psychology, 8, 765-777.

Zeiss, A. M., Lewinsohn, P. M. y Muñoz, R. F. (1979). Nonspecific improvement effects in depression using interpersonal cognitive, and pleasent events focused treatments. Journal of Consulting and Clinical Psychology, 47, 427-439.

Manuscrito recibido: 03/09/2009

Revisión recibida: 01/10/2009

Manuscrito aceptado: 28/10/2010 